\title{
STRONGLY $\pi$-REGULAR MATRIX SEMIGROUPS
}

\author{
JAN OKNIŃSKI
}

\begin{abstract}
We prove that if $S$ is a strongly $\pi$-regular multiplicative subsemigroup of the matrix algebra $M_{n}(K), K$ being a field, then there exists a chain of ideals $S_{1} \triangleleft \cdots \triangleleft S_{t}=S$ such that $t \leq 2^{n+1}$ and any Rees factor semigroup $S_{i} / S_{i-1}$ is either completely 0-simple or nilpotent of index not exceeding $\prod_{j=0}^{n}\left(\begin{array}{c}n \\ j\end{array}\right)$. This sharpens the main result of $[4]$, in particular solving Problem 3.9 from $[\mathbf{3}]$.
\end{abstract}

A semigroup $S$ is said to be strongly $\pi$-regular if for any $s \in S$ there exists an integer $k \geq 1$ such that $s^{k}$ lies in a subgroup of $S$. Matrix semigroups (i.e. multiplicative subsemigroups of the matrix algebra $M_{n}(K)$ for some field $K$ and some $n \geq 1$ ) of this type have been recently the suject of considerable study (for references see [4]). In particular, Putcha proved that any such semigroup admits a chain of ideals $S_{1} \triangleleft \cdots \triangleleft S_{t}=S$ with all factor semigroups $S_{i} / S_{i-1}$ being completely 0 -simple or nilpotent [4]. The purpose of this note is to show that, in this case, $t$ cannot exceed $2^{n+1}$ and that there is a bound (also depending on $n$ only) on indices of nilpotency of the nilpotent factors. The former solves Problem 3.9 from [3]. The way we shall proceed is quite different from that used in [4] where the Zariski closure was the main tool.

If $a \in M_{n}(K)$, then by $\rho(a)$ we shall denote the rank of $a$. Further, for any $j$, $1 \leq j \leq n$, we put $I_{j}=\left\{a \in M_{n}(K) \mid \rho(a) \leq j\right\}$. Certainly, $I_{j}$ is an ideal of the semigroup $M_{n}(K)$. Let $a \in M_{n}(K)$. Then, treating $a$ as a linear transformation of an $n$-dimensional vector space over $K$ with a fixed basis, we shall denote by $\Lambda^{j} a$ the $j$ th exterior power of $a$ and treat it (in a usual way) as an element of $M_{\left(\begin{array}{c}n \\ j\end{array}\right)}(K)$.

We are indebted to Dr. Z. Marciniak for bringing the following well-known result as well as its usefulness for our considerations to our attention.

LEMMA 1 (CF. [1, §5, EXeRCiSe 11]). Let $1 \leq j \leq n$. Then $\Lambda^{j}: M_{n}(K) \rightarrow$ $M_{\left(\begin{array}{c}n \\ j\end{array}\right)}(K)$ is a semigroup homomorphism. Moreover $\rho\left(\Lambda^{j}(a)\right)=0$ if $\rho(a)<j$ and $\rho\left(\Lambda^{j}(a)\right)=\left(\begin{array}{c}\rho(a) \\ j\end{array}\right)$ if $\rho(a) \geq j$.

We shall start with an auxiliary result concerning matrices of rank one.

LEMMA 2. Let $T \subset M_{n}(K)$ be a set of idempotents of rank one. Then:

(1) if $(e f)^{2}=0$ for any $e, f \in T, e \neq f$, then $|T| \leq 2^{n}-1$,

(2) if $(e f g)^{2}=0$ for any $e, f, g \in T, e \neq g$, then $|T| \leq n$.

PROOF. We will proceed by induction on $n$. The result is obvious if $n=1$. Let $n>1$ and $f, g \in T, f \neq g$. Since $\rho(g)=\rho(f)=1$, the condition $(f g)^{2}=0$ easily implies that $f g=0$ or $g f=0$. Thus, in any case, $(1-g) f(1-g)$ is an idempotent.

Received by the editors March 23, 1984.

1980 Mathematics Subject Classification. Primary 20M10. 
Let us fix $e \in T$ and define $\bar{f}=(1-e) f(1-e)$ for $f \in T \backslash\{e\}, \bar{T}=\{\bar{f} \mid f \in T \backslash\{e\}\}$. Then $\bar{T}$ embeds into $M_{n-1}(K)$. Since $\bar{f}=0$ would imply $e f=f$ or $f e=f$, then by hypothesis $\rho(\bar{f})=1$ for any $\bar{f} \in \bar{T}$.

(1) Put $T_{1}=\{f \in T \mid e f=0\}, T_{2}=\{f \in T \mid f e=0\}$ and $\bar{T}_{i}=\left\{\bar{f} \mid f \in T_{i}\right\}$ for $i=1,2$. If $f, g \in T_{1}$, then $(\bar{f} \bar{g})^{2}=(f g)^{2}(1-e)$. Hence $(\bar{f} \bar{g})^{2}=0$ if and only if $f \neq g$. This means that $\bar{T}_{1}$ satisfies the induction hypothesis and $\left|\bar{T}_{1}\right|=\left|T_{1}\right|$. Since similar arguments can be applied to the set $T_{2}$, by the induction argument we then get

$$
|T| \leq\left|T_{1}\right|+\left|T_{2}\right|+1=\left|\bar{T}_{1}\right|+\left|\bar{T}_{2}\right|+1=2\left(2^{n-1}-1\right)+1=2^{n}-1 .
$$

(2) It may be easily checked that for any $\bar{f}, \bar{g} \in \bar{T}, f \neq g$, the element $\bar{f} \bar{g}$ must be nilpotent. For example, if $e f=f g=g e=0$, then $(\bar{f} \bar{g})^{2}=(f e g)^{2}=0$. Hence, in particular, $\bar{f}=\bar{g}$ implies $f=g$. Thus, as above, the inclusion argument yields $|T|=|\bar{T}|+1 \leq n$.

It is easy to see that the assumption of (1) in Lemma 2 is essentially weaker than that of (2). In fact, take, for example,

$$
T=\left\{\left(\begin{array}{ll}
1 & 0 \\
0 & 0
\end{array}\right),\left(\begin{array}{ll}
0 & 0 \\
1 & 1
\end{array}\right),\left(\begin{array}{cc}
0 & 1 \\
0 & -1
\end{array}\right)\right\} \subset M_{2}(K) .
$$

COROLlarY 1. Let $T \in M_{n}(K)$ be a set of idempotents of rank $j$. Then

(1) if $\rho\left((e f)^{2}\right)<j$ for any $e, f \in T, e \neq f$, then $|T| \leq 2\left(\begin{array}{l}n \\ j\end{array}\right)-1$,

(2) if $\rho\left((e f g)^{2}\right)<j$ for any $e, f, g \in T, e \neq g$, then $|T| \leq\left(\begin{array}{l}n \\ j\end{array}\right)$.

Proof. Let us consider the set $\Lambda^{j}(T) \subset M_{\left(\begin{array}{c}n \\ j\end{array}\right)}(K)$. Since $\Lambda^{j}$ is a homomorphism, then the assumption of (1) (hence, also the assumption of (2)) implies that $|T|=\left|\Lambda^{j}(T)\right|$. Moreover, by Lemma $1, \Lambda^{j}(T)$ consists of idempotents of rank one satisfying the hypotheses of Lemma 2(1), (2) accordingly. Thus, the result follows from Lemma 2.

The first part of Corollary 1 solves Problem 3.9 from [3]. However, to get a stronger result in our structure theorem we will use the second assertion of Corollary 1.

Proposition 1. Let $S \subset M_{n}(K)$ be a strongly $\pi$-regular semigroup. Then $S$ has at most $2^{n}$ regular J-classes.

Proof. Let $1 \leq j \leq n$. Assume that some idempotents $e, f, g \in S$ of rank $j$ are given with $e, g$ lying in distinct $J$-classes. Suppose that $\rho\left((e f g)^{2}\right)=j$. Then, since $S$ is strongly $\pi$-regular, there exist $x \in S$ and $y=y^{2} \in S$ such that $(e f g)^{k} x=y$ and $\rho(y)=\rho\left((e f g)^{k}\right)$ for some $k \geq 1$. Thus $\rho(y)=j$. Now $y=y^{2}=(e y)^{2}$ and so $\rho($ eye $) \geq \rho(y)=j$ which implies that $\rho($ eye $)=j$. Since $(\text { eye })^{2}=$ eye, this yields $e y e=e$. Hence $(e f g)^{k} x e=e y e=e$ and so $e \in S g S$. Similarly $g \in S e S$, a contradiction. Now, from Corollary 1 it follows that there are at most $\left(\begin{array}{l}n \\ j\end{array}\right)$ regular $J$-classes of $S$ contained in $I_{j} \backslash I_{j-1}$. This yields the result.

Let us observe that there exists a semigroup $T \subset M_{n}(K)$ which is strongly $\pi$ regular and has exactly $2^{n}$ regular $J$-classes-namely the set of all diagonal idempotents. 
PROPOSITION 2. Let $S \subset M_{n}(K)$ be a strongly $\pi$-regular semigroup. If $I$ is an ideal of $S$ such that $S / I$ is a nil semigroup, then $(S / I)^{m}=0$, where $m=\prod_{j=1}^{n}\left(\begin{array}{c}n \\ j\end{array}\right)$.

Proof. Let $j$ be the least integer such that $S \subset I_{j}$. Define $H$ as the subsemigroup generated by $S \backslash I$. Let $h=g_{1} \cdots g_{s}, g_{i} \in S \backslash I$. By hypothesis, there exist $y \in S, e=e^{2} \in S, k \geq 1$, such that $h^{k} y=\left(g_{1} \cdots g_{s}\right)^{k} y=e$ and $\rho(e)=\rho\left(h^{k}\right)$. Suppose that $\rho(e)=j$. Then $\rho(h)=\rho(e)$ and from [4, Lemma 4], it follows that $g_{1}=e g_{1}$. Since $I$ is an ideal of $S$, then we must have $e \notin I$. This contradicts the fact that $S / I$ is nil. Hence $\rho\left(h^{k}\right)=\rho(e)<j$. While $h \in H$ is an arbitrary element, $H /\left(H \cap I_{j-1}\right)$ is a nil semigroup.

Let us consider the semigroup $\Lambda^{j}(H) \subset M_{\left(\begin{array}{c}n \\ j\end{array}\right)}(K)$. Since, by Lemma $1, \Lambda^{j}\left(I_{j-1}\right)$ $=0$, then the first part of the proof implies that $\Lambda^{j}(H)$ is a nil semigroup. Thus, it is well known that $\Lambda^{j}\left(H^{\left(\begin{array}{c}n \\ j\end{array}\right)}\right)=\Lambda^{j}(H)^{\left(\begin{array}{c}n \\ j\end{array}\right)}=0$ [2, Proposition 17.19]. This means that $H^{\left(\begin{array}{l}n \\ j\end{array}\right)} \subset I_{j-1}$. Now we have a natural epimorphism $H /\left(H \cap I_{j-1}\right) \rightarrow$ $S /\left(I \cup\left(I_{j-1} \cap S\right)\right)$ and the latter is also nilpotent of index not exceeding $\left(\begin{array}{l}n \\ j\end{array}\right)$. On the other hand

$$
\left(I \cup\left(I_{j-1} \cap S\right)\right) / I \simeq\left(I_{j-1} \cap S\right) /\left(I \cap I_{j-1} \cap S\right)=\left(I_{j-1} \cap S\right) /\left(I_{j-1} \cap I\right) .
$$

Putting $\bar{S}=I_{j-1} \cap S, \bar{I}=I_{j-1} \cap I$, we get a strongly $\pi$-regular semigroup $\bar{S} \subset I_{j-1}$ and we may repeat the above procedure regarding the nil semigroup $\bar{S} / \bar{I}$. Thus,

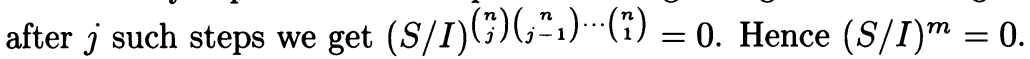

Let us notice that Proposition 2 provides in particular an alternative proof of the implication $S / I$-nil $\Rightarrow S / I$-nilpotent, which was proved in [4].

Now, by Propositions 1 and 2 and the structure theorem for strongly $\pi$-regular semigroups with finitely many regular J-classes (cf. [3, Lemma 1.9]) we can summarize our results.

THEOREM. Let $S \subset M_{n}(K)$ be a strongly $\pi$-regular semigroup. Then there exists a chain of ideals $S_{1} \triangleleft \cdots \triangleleft S_{t}=S$ such that $t \leq 2^{n+1}$ and all factors $S_{i} / S_{i-1}$ are completely 0 -simple or nilpotent of index not exceeding $m=\prod_{j=1}^{n}\left(\begin{array}{c}n \\ j\end{array}\right)$.

At last, observe that, in view of [4], the Theorem establishes the nonexistence of chains of left (right) principal ideals of $S$ with length exceeding $m^{2^{n+1}}, m$ as above.

ADDED IN PROOF. Since any periodic semigroup $S$ is strongly $\pi$-regular, the Theorem provides a simple proof of the Burnside theorem for semigroups (cf. [5]). In fact, to show that $S$ is locally finite it is enough to prove that all $S_{i} / S_{i-1}$ are locally finite (cf. [6, Lemma 3]). While the nilpotent case is obvious, the completely 0-simple case easily follows by Rees' theorem and the Burnside theorem for torsion groups.

\section{REFERENCES}

1. N. Bourbaki, Algèbre, Chapitre III, Hermann, Paris, 1948.

2. C. Faith, Algebra. II. Ring theory, Springer-Verlag, Berlin and New York, 1976.

3. M. Putcha, On algebraic linear semigroups, Trans. Amer. Math. Soc. 259 (1980), 457-469.

4. __ Matrix semigroups, Proc. Amer. Math. Soc. 88 (1983), 386-390.

5. R. McNaughton and Y. Zalcstein, The Burnside problem for semigroups, J. Algebra 34 (1975), 292-299.

6. J. Okniński, On regular semigroup rings, Proc. Roy. Soc. Edinburgh (to appear). 